International Journal of Research Studies in Computer Science and Engineering (IJRSCSE)

Volume 6, Issue 2, 2019, PP 18-23

ISSN 2349-4840 (Print) \& ISSN 2349-4859 (Online)

DOI: http://dx.doi.org/10.20431/2349-4859.0602003

www.arcjournals.org

\title{
Carrier Aggregation based Downlink Packet Scheduling Scheme with Improved Quality of Service
}

\author{
Durga Devi Thulluri ${ }^{*}$, Someswari Perla², Yugandhar Manchala ${ }^{3}$ \\ ${ }^{1}$ Asst. prof, CSE Department, RISE krishna Sai Prakasham Group of institutions, Ongole. \\ ${ }^{2}$ Asst. Prof, CSE Department, GMR Institute of Technology, Rajam \\ ${ }^{3}$ Asst. Prof, CSE Department, Aditya Institute of Technology and Management, Tekkali.
}

*Corresponding Author: Durga Devi Thulluri, RISE krishna Sai Prakasham Group of institutions, Ongole.

\begin{abstract}
Carrier Aggregation (CA) is one of the important features to support wider bandwidth for transmitting the packets in Long Term Evolution (LTE-A) Systems. Though there has been considerable work existing on Carrier Aggregation based packet scheduling, to the best of our knowledge the work regarding CA based LTE-A system packet scheduling considering service differentiation has not been investigated thoroughly till now. This paper focuses on packet scheduling using two methods, the first a conventional water filling method which does not consider service classes and the other one that considers the service class for packet aggregation. We consider downlink transmission which supports both Real-Time (RT) and non-Real-Time traffic simultaneously. Simulation results shows that while the water filling method indeed provides better performance in terms of delays in LTE system, the Service Class Based Carrier Aggregation(SCBCA) improves the throughut and provides better fairness among the service flows of different classes.
\end{abstract}

Keywords: Carrier Aggregation, LTE-A, RT, NRT, WF

\section{INTRODUCTION}

To achieve the capacity of present cellular network with fast growing data demand in the International Telecommunication Union has defined the specification of 4G systems. 4g systems support high system capacity. They are also called as International Mobile Telecommunications-Advanced (IMT-A) cellular systems

Based on the economic and service demands IMT-A systems must provide their support to the data rates. In the 3rd Generation Partnership Project (3GPP), it is agreed that Long Term EvolutionAdvanced (LTE-A) systems, the evolution version of LTE systems, should meet the requirement of IMT-A systems. The progress of key technologies in LTE-A and IMT-A systems can be referred in [1][2].

LTE-Advanced should support wider bandwidth for high peak data rates and wide coverage. Carrier aggregation

(CA),a technique that aggregates multiple adjacent component carriers into an overall wider bandwidth, is preferred for the spectrum compatibility.

To Design an ideal packet scheduler in LTE-A system with CA the requirements to be satisfied are: 1) Requires to handle the packet scheduling in multiple CCs environments, 2) the required Quality-ofService (QoS) needs to be supported for various kinds of traffics, 3) requires to achieve high system throughput, and 4) the fairness among users, traffic classes must be maintained.

Many researchers have started to investigate the issues related to design of the scheduler for the LTEA system with carrier aggregation, like L. Lei and K. Zheng analysed both the delay and the throughput performance in the LTE-A systems with carrier aggregation mechanism. L. Chenet al and K. Takeda et al. had proposed several CCs scheduling algorithms and scheduler structures in [3]. To evaluate the performance of independent carriers in LTE-Advanced systems, several scheduling algorithms and scheduler structures are proposed. Performance results are compared with independent carrier(IC) mechanism, where CCs are not aggregated but used independently.

The Purpose of this paper is to study the system level performance of CA in LTE-Advanced systems with and without considering service traffic class requirements.

International Journal of Research Studies in Computer Science and Engineering (IJRSCSE) Page 18 


\section{SYSTEM MODEL}

In this paper we considered LTE-A system in a single cell environment and we focus on down link transmission. It consists of $\mathrm{n}$ user terminals (both RT and NRT) and a Base Station (BS). Here we assume that there exist $\mathrm{c}$ adjacent $\mathrm{CCs}$ in the same frequency bandwidth aggregated in a single BS.

Considering all CCs have the same bandwidth, the $\mathrm{i}^{\text {th }} \mathrm{CC}$ has $\mathrm{b}_{\mathrm{i}}$ Resource Blocks (RBs), where $\mathrm{RB}$ is smallest allocation unit for resource scheduling. Hence, CA contains $b_{\text {total }}=\Sigma^{c}{ }_{i=1} b_{i} R B$ s available for packet transmission. Assume that each RB contains the same transmission power.

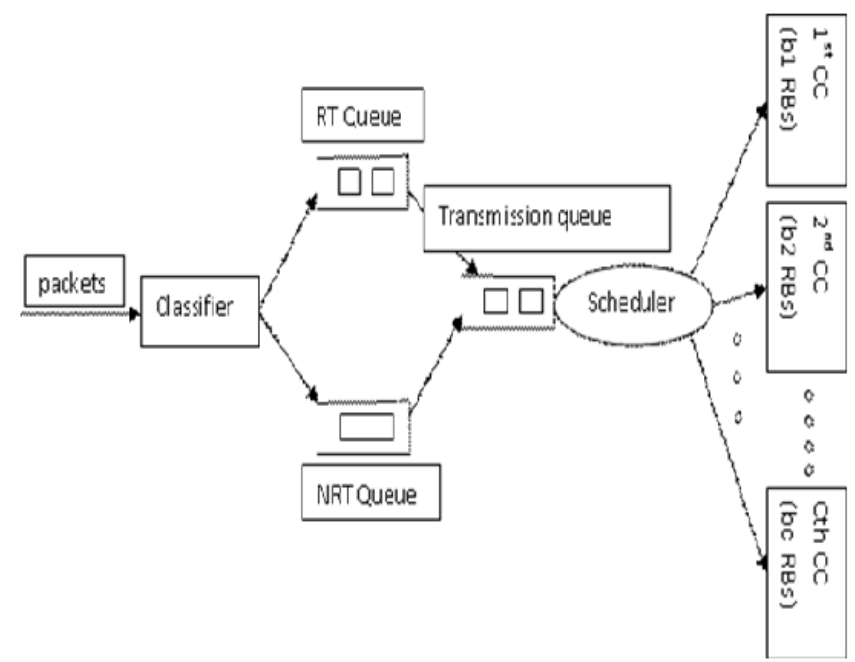

Figure1. The system model of a classifier with RT queue and NRT, transmission queue and CCs with $i^{\text {th }} C C$ containing $b_{i} R B s, i=1,2,3 \ldots \ldots ., c$, in a downlink transmission

This figure explains that, data received by the classifier in the form of packets which is divided into the RT packets and NRT packets. This data will be delivered into RT queue and NRT queue, respectively, based on FCFS discipline. The packets in the RT queue and NRT queue will be scheduled on the basis of the proposed packet scheduling algorithm used in scheduler, to the CCs for parallel transmissions simultaneously. The existing packet scheduling algorithm is based on PF criteria. We define $\delta_{\mathrm{RT}}$ as a delay constraint for each RT packet.

Here, Orthogonal Frequency Division Multiplexing Access (OFDMA) radio technology is considered as downlink radio access scheme because of some special features like spectral efficiency, bandwidth scalability and robustness against the multi-path fading [4]. The duration of an OFDMA downlink frame can be denoted as tOFDMA used in a run for scheduling packets. The current run can be denoted by the symbols.

\subsection{Packet Scheduling Algorithms}

In this algorithm we briefly elaborate about PF[5] criterion for packet scheduling. Next we will present efficient packet scheduling algorithm based on that Dead line Service Class Based Carrier Aggregation (SCBCA).

\subsubsection{Proportional Fair Criteria}

The PF utility function indicates the ratio of the users instantaneous data rate to average data rate. For scheduling, the first chance will be given to the user who has the highest PF factor.

$k^{*}=\arg \max \frac{R_{k}}{R_{k}^{*}}$

PF scheme is mostly suitable for NRT traffics, it achieves much larger throughputs than other respective packet scheduling schemes like Round Robin(RR), Deficit Round Robin(DRR). The objective of existing algorithm is to optimize the overall system throughput as much as possible by maintaining fairness among all users and required Quality of Service (QoS) of RT traffic.

The NRT packet in NRT queue will be delivered to the transmission queue periodically. Each period length denoted as $t_{t h}$, it is an integral multiple of run from first run. The RT packets in RT queue will be delivered to the transmission queue run-by-run. 
$\eta=b_{N R T} / b_{\text {total }}$, is number of RBs that NRT users can share to $b_{\text {total }}$ to the level $\eta$. $b_{\mathrm{NRT}} \epsilon$ $\left\{1,2,3, \ldots \ldots \ldots, b_{\text {total }}\right\}$. Here RB is a sequence on CC starts with index $i=1$.NRT packets can be transmit over $\eta b_{\text {total }}$ RBs while RT packet can be transmit over $b_{\text {total }}$ RBs. RBs are always reserved only for RT packets.

In existing algorithm for each run, the first chance will be given for NRT packet transmission and then RT packet delivery to transmission queue from corresponding queues. Once the RT packet transmission violates threshold $\delta_{\mathrm{RT}}$ condition, stop the processing of RT packet transmission, store the data into CC starting with unused RBs and calculate the fairness vector by using CCs, RBs and number of users, for giving priority to the user.

$$
\operatorname{tuple}\left(i^{*}, j^{*}, k^{*}\right)=\arg \max _{i, j, k} \frac{r_{k}(i, j, s)}{r_{k}^{*}}
$$

If the size of the packet is larger than the available size of PRB, then the packet is split, the first part is stored in first PRB and the next part is stored in next PRB. The average data rate for each user is updated on every run, by using the formula

$r_{k}^{*}=\left(1-\frac{1}{\tau}\right) r_{k}^{*}+\frac{1}{\tau} r_{k}(i, j, s)$

Where " $\tau$ " is a constant value for averaging the user k's data rate.

If there exists any remaining resources the same procedure repeats once again until the completion of all resources.l

\subsubsection{The Water Filling Model}

Intuitively, in the conventional water filling model, non-real time users may be given higher priority, which may deprive the real time users of their service time requirements. However, if we neglect nonreal time users during scheduling, their throughput requirements may not be met either. Moreover, the method proposed in 2.1.1 may at times favor non-real time flows to real-time flows owing to the backlogged non-real time queues. The suggested method Service Class Based Carrier Aggregation(SCBCA) attempts to correct the situation by giving RT users greater opportunities, while at the same time ensuring that a fraction of opportunity is given to non-real time flows which effectively avoids backlogging of the queues. This obviously may mean some effect on delay of real time flows, but it was observed to vastly improve the System throughput and fairness among traffic classes.

\subsection{Service Class Based Carrier Aggregation}

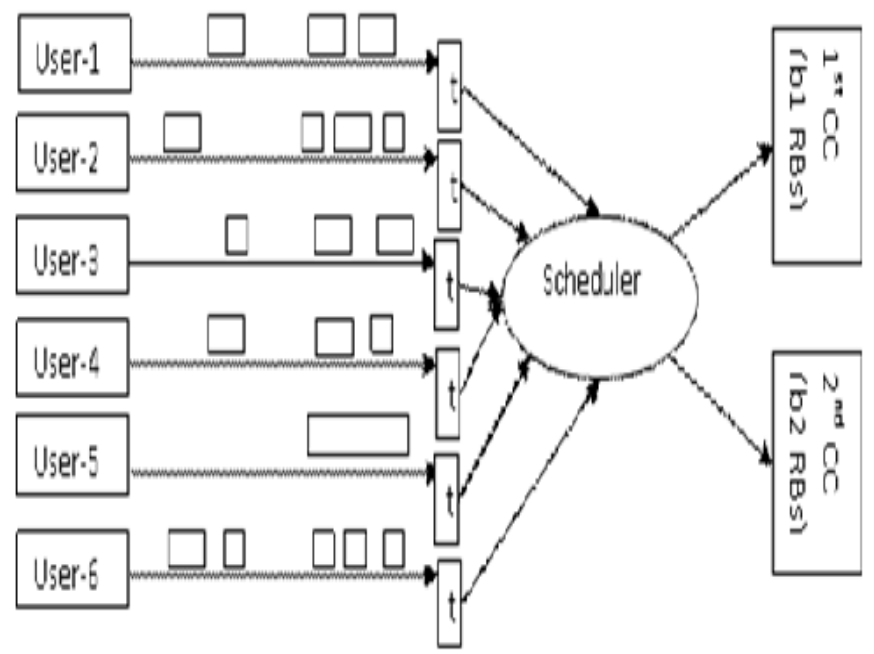

Figure2. Downlink transmissions considering 2 CCs aggregated in a BS and 6 users including some RT and some NRT.

Even though the user has higher priority traffic, he may suffer as a result frequent backlogging of nonreal time queues. To avoid this we consider,

- A particular amount of time period is fixed for each user

- Same time period will be given for all users.

International Journal of Research Studies in Computer Science and Engineering (IJRSCSE) 
- So the scheduler receives the packets from users based on time lines.

- The first chance will be given for RT users.

- Scheduler will receive the Users packets and schedule them to respective CCs.

- Few CCs are reserved for RT-users in the same way for NRT-users too, based on requirement

- Once the first run is completed it checks for availability of packets.

- If the packets are available, the same process will

- be continued until the completion of all packets.

- The same procedure will be applicable for the next coming runs too.

- If any packet size is larger than PRB size, then packet is partitioned first part is stored in first PRB and the later part is stored in next PRB.

\subsection{Baseline Scheduler}

The main principle of the baseline scheduling algorithm is that the multiple CCs cannot be used independently. Each RT user will be randomly assigned to only one particular CC among all CCs assigned for RT-users for packet transmission. Meanwhile each NRT-user will randomly assigned to only one particular CC assigned for NRT-users.

\section{Simulation Results}

In this section we examine the long-term system throughput, delay and fairness among the users. Here we are assuming that coverage capacity is $1 \mathrm{~km} .2$ adjacent CCs in the $2 \mathrm{GHz}$ frequency band are taken to be aggregated in the BS for packet transmission. Data transmission rate is considered as $1000 \mathrm{Mbps}$.

Each CC contains 25 PRB's. The bandwidth of CC is set to be 5MHz. Each CC contains 512 subcarriers and FFT size is set as 512. Each PRB bandwidth is fixed as $180 \mathrm{MHz}$. The transmitting speed is of every RT user is assumed as $3 \mathrm{~km} / \mathrm{hr}$ of uniform distribution in the random direction. NRT users can access the network in a fixed location. $t_{\mathrm{th}}$ and $\delta_{\mathrm{RT}}$ can be considered as 3 and 20 runs, respectively.

For demonstrating the simulation results, all user traffics are independently generated according to the ON-OFF model. The OFF duration for RT and NRT packets are with distribution mean 0.03 and 0.05 seconds respectively. The ON duration for RT and NRT packets are with distribution mean 0.01 and 0.1 seconds, respectively. While ON the packet size for Rt and NRT packets are generated with a truncated geometric distribution with the mean 100 and 300 bytes, respectively. The modified COST231 Hata model [7] is considered for the path-loss model which is widely used for predicting the pathloss in several mobile wireless systems. The path-loss model for urban macro-cell at the frequency

$$
f(2 \mathrm{GHz} \leq f \geq 6 \mathrm{GHz}) \text { is expressed by }
$$

$\mathrm{PL}_{\text {urban-macro }}[\mathrm{dB}]=35.2+35 \log (\mathrm{d})+26 \log (f / 2)$, where $\mathrm{d}$ is the distance from the $\mathrm{BS}$ to the user.

\subsection{Throughput Comparison}

Here we are comparing the throughput of both Water Filling algorithm (WF) and Service Class Based Carrier Aggregation in Fig 3.

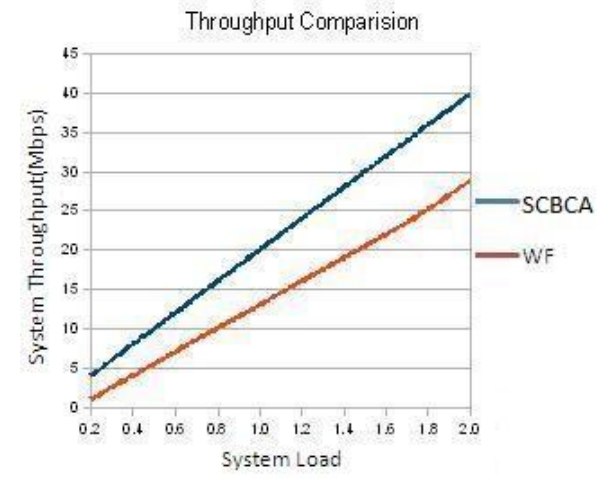

Figure3. The system throughput comparison between WF and SCBCA versus the System Load. 
The system Load can be defined as the ratio of the total packet arrival rate to the maximum system service rate. The maximum system service rate can be considered as $14.8 \mathrm{Mbps}$. Here the system load is depends upon the packet

\subsection{Mean Packet Delay Comparison}

Here we are examining the mean packet delay versus system load. Packet delay means the time measured starting from the packet arrived into classifier until the packet is completely transmitted by the OFDMA frame.

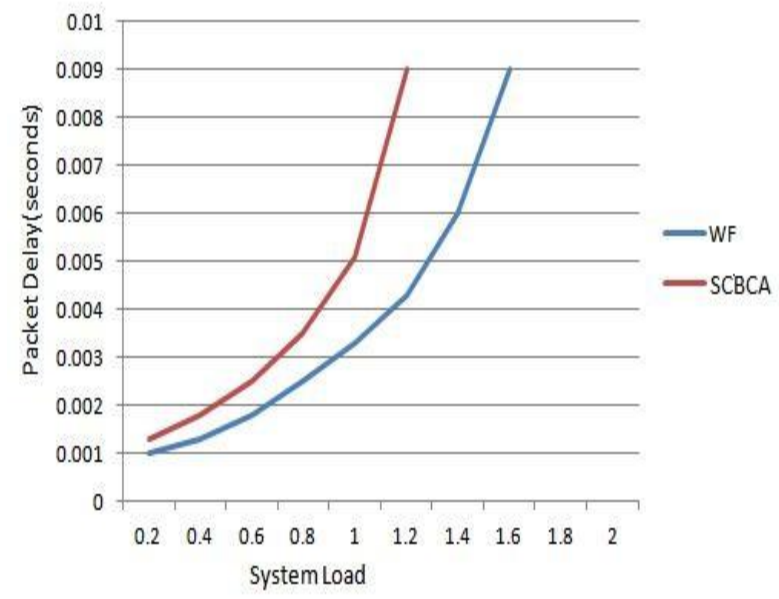

Figure4. The mean packet delay comparison of RT packets between WF and SCBCA versus system load.

The RT packet is lesser the than NRT packet delay for both the algorithm, though SCBCA has larger packet delays at higher load compared to WF scheduling.

\subsection{Fairness Comparison}

For completeness, the fairness of WF and SCBCA is evaluated. Here we are using R. Jain et al.'s fairness index [6] can be defined as

$F=\frac{\left(\sum_{k=1}^{n} r_{k}\right)^{2}}{\left(\sum_{k=1}^{n}\left(r_{k}\right)^{2}\right)}$

to quantify the fairness among all users. This graph shows the system load versus fairness index of both WF and SCBCA. Fairness index of SCBCA is slightly better than WF. It is because of each RT flows are scheduled with equal opportunities compared to the WF method. The fairness is almost equals to 1 when the system load is low.

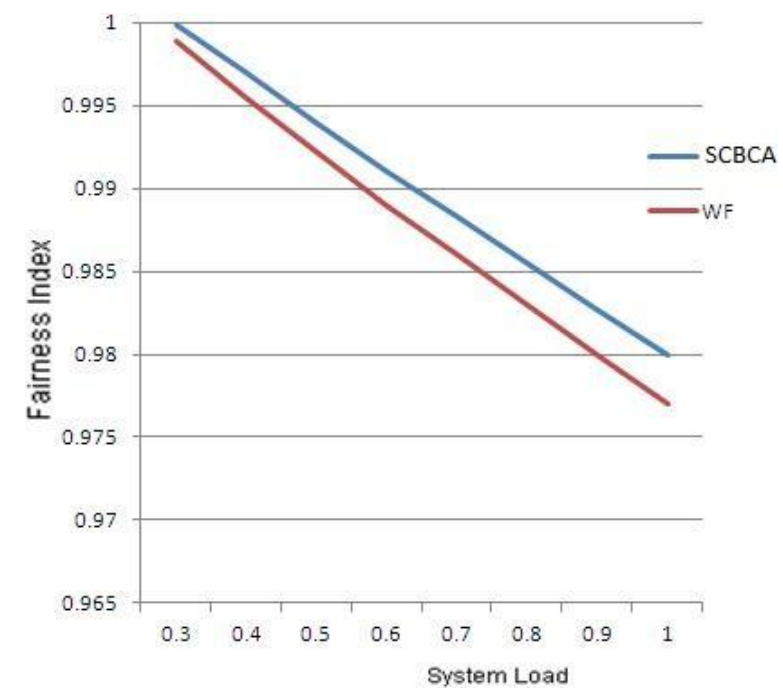

Figure5. The Fairness Index comparison of RT packets between WF and SCBCA versus system load 


\section{CONCLUSION}

Carrier Aggregation based Downlink Packet Scheduling Scheme with improved throughput has successfully been proposed for LTE-A systems. By adjusting with $\eta$ and $t_{\text {th }}$ we can achieve high QoS for both RT and NRT traffics. Mean while we can achieve fairness among all users by using SCBCA criteria by following PF[8].From simulation results, the SCBCA achieves significant improvements in the system throughput and the fairness when compared with the case without WF method.

To summarize, timely delivery of RT traffics and NRT traffics at high throughput are well supported by SCBCA criteria, since all CCs are flexibly and effectively utilised. The proposed scheme is useful to consider the real world traffic patterns with various kinds of quality-of-service requirements for downlink transmissions in LTE-A systems.

\section{ACKNOWLEDGEMENT}

I convey my sincere thanks to, Dr. C. Kalyana Chakravarthy, professor, CSE Dept, for his immense help during this project.

\section{REFERENCES}

[1] S. Parkwall and D. Astely, "The evolution of LTE towards IMT-advanced" journal of communications. Vol 4, no. 3, April 2009.

[2] D. Martin-Sacristan et al.," On the way towards fourth generation mobile: 3GPP LTE and LTE-Advanced “, EURASIP journal on wireless communication and networking, vol.209, Article ID354089, 10 pages, 2009.

[3] L. Chen et al, "Analysis and simulation for spectrum aggregation in LTE-advanced system" IEEE VTC 2009 Fall, Anchorage, Alaska, USA, 2009.

[4] [4] S. B. Lee et al" Downlink MIMO with frequency domain packet scheduling for 3GPP LTE ”, 2009. Rio de Janeiro, Brazil 2009.

[5] F. Kelly et al, "Rate control for communication networks: shadow prices, proportional fairness and stability,”-_RI WKH2SHUDWLRQDO 5HVHDUFK 6RFLHW, vol. 49,pp. 237-252,Apr 1998.

[6] R. Jain et al, "A Quantitative measure of fairness and discrimination for resource allocation in shared computer systems",DEC-TR-301, Sept 26, 1984.

[7] COST Action 231, “ Digital mobile radio towards future generation systems, final reports," Tech. Rep., European Communities, EUR 18957, 1999.

[8] Jalali et al.," Data throughput of CDMA-HDR a high efficiency-high data rate personal communication wireless system," IEEE VTC 2000 Spring, Tokyo, Japan, 2000.

[9] Yao-Liang Chung, Lih jong jang," An Efficient Downlink Packet Scheduling Algorithm in LTE-Advanced Systems with Carrier Aggregation"

Citation: Durga Devi Thulluri, et.al., (2019). Carrier Aggregation based Downlink Packet Scheduling Scheme with Improved Quality of Service. International Journal of Research Studies in Computer Science and Engineering (IJRSCSE), 6(2), pp.18-23. http://dx.doi.org/10.20431/2349-4859.0602003

Copyright: () 2019 Authors, this is an open-access article distributed under the terms of the Creative Commons Attribution License, which permits unrestricted use, distribution, and reproduction in any medium, provided the original author and source are credited. 